\title{
Heat Transfer between Weakly Coupled Systems: Graphene on a-SiO 2
}

\author{
B.N.J. Persson ${ }^{1,2}$ and H. Ueba ${ }^{1}$ \\ 1 Division of Nanotechnology and New Functional Material Science, \\ Graduate School of Science and Engineering, University of Toyama, Toyama, Japan and \\ ${ }^{2}$ IFF, FZ-Jülich, 52425 Jülich, Germany, EU
}

\begin{abstract}
We study the heat transfer between weakly coupled systems with flat interface. We present a simple analytical result which can be used to estimate the heat transfer coefficient. As an application we consider the heat transfer between graphene and amorphous $\mathrm{SiO}_{2}$. The calculated value of the heat transfer coefficient is in good agreement with the value deduced from experimental data.
\end{abstract}

Almost all surfaces in Nature and Technology have roughness on many different length scales[1]. When two macroscopic solids are brought into contact, even if the applied force is very small, e.g., just the weight of the upper solid block, the pressure in the asperity contact regions can be very high, usually close to the yield stress of the (plastically) softer solid. As a result good thermal contact may occur within each microscopic contact region, but owing to the small area of real contact the (macroscopic) heat transfer coefficient may still be small. In fact, recent studies have shown that in the case of surfaces with roughness on many different length scales, the heat transfer is independent of the area of real contact [2]. We emphasize that this remarkable and counter-intuitive result is only valid when roughness occur over several decades in length scale.

For nanoscale systems the situation may be very different. Often the surfaces are very smooth with typically nanometer (or less) roughness on micrometer-sized surface areas, and because of adhesion the solids often make contact over a large fraction of the nominal contact area. The heat transfer between solids in perfect contact is usually calculated using the so called diffusive mismatch model [3] , where it is assumed that all phonons scatter diffusively and elastically at the interface between two materials. In this model there is no direct reference to the nature of the solid-solid interaction accross the interface, and the model cannot describe the heat flow between weakly interacting solids.

Here we will discuss the heat transfer across perfectly flat interfaces, when the interaction between the solids is very weak, e.g., of the Van der Waals type, as for graphene or carbon nanotubes on many substrates. We present a simple analytical result which can be used to estimate the heat transfer coefficient. As an application we consider the heat transfer between graphene and amorphous $\mathrm{SiO}_{2}$. The calculated value of the heat transfer coefficient is in good agreement with the value deduced from experimental data.

Consider the interface between two solids, and assume that local thermal equilibrium occurs everywhere except at the interface. The energy flow (per unit area) through the interface is given by [2]

$$
J=\alpha\left(T_{0}-T_{1}\right)
$$

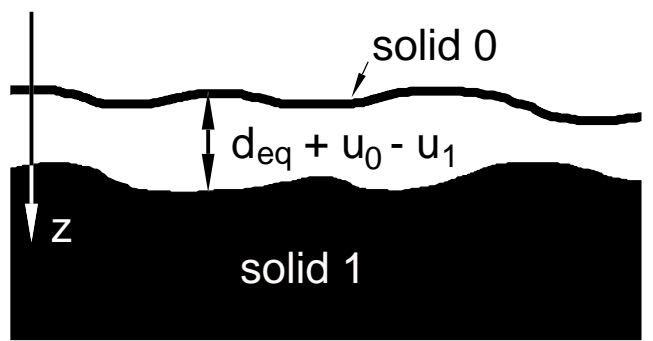

FIG. 1: A membrane (solid $\mathbf{0}$ ) in contact with a semi-infinite solid 1. The interfacial surface separation is the sum of the equilibrium separation $d_{\mathrm{eq}}$ and the difference in the surface displacements $u_{0}-u_{1}$, due to thermal movements, where both $u_{0}$ and $u_{1}$ are positive when the displacement point along the $z$-axis towards the interior of solid $\mathbf{1}$.

where $T_{0}$ and $T_{1}$ are the local temperatures at the interface in solid $\mathbf{0}$ and $\mathbf{1}$, respectively. We now present a calculation of the heat transfer coefficient $\alpha$ under the assumption of very weak coupling between the two solids.

The stress or pressure acting on the surface of solid $\mathbf{1}$ from solid $\mathbf{0}$ can be written as

$$
\sigma(\mathbf{x}, t)=K\left[u_{0}(\mathbf{x}, t)-u_{1}(\mathbf{x}, t)\right],
$$

where $u_{0}$ and $u_{1}$ are the (perpendicular) surface displacement of solid $\mathbf{0}$ and $\mathbf{1}$ (see Fig. 11), respectively, and where $K$ is a spring constant per unit area characterizing the interaction between the two solids. If we define

$$
u(\mathbf{q}, \omega)=\frac{1}{(2 \pi)^{3}} \int d^{2} x d t u(\mathbf{x}, t) e^{-i(\mathbf{q} \cdot \mathbf{x}-\omega t)},
$$

we get

$$
\sigma(\mathbf{q}, \omega)=K\left[u_{0}(\mathbf{q}, \omega)-u_{1}(\mathbf{q}, \omega)\right] .
$$

If solid $\mathbf{1}$ is semi-infinite we have[4]

$$
u_{1}(\mathbf{q}, \omega)=M(\mathbf{q}, \omega) \sigma(\mathbf{q}, \omega),
$$

where $M(\mathbf{q}, \omega)$ is determined by the elastic properties of solid 1. Combining (1) and (2) gives

$$
u_{1}(\mathbf{q}, \omega)=\frac{K M(\mathbf{q}, \omega)}{1+K M(\mathbf{q}, \omega)} u_{0}(\mathbf{q}, \omega)
$$


The energy transferred to solid $\mathbf{1}$ from solid $\mathbf{0}$ during the time period $t_{0}$ can be written as

$$
\Delta E=\int d^{2} x d t \dot{u}_{1}(\mathbf{x}, t) \sigma(\mathbf{x}, t)
$$

where $\dot{u}=\partial u / \partial t$. One can also write

$$
\Delta E=(2 \pi)^{3} \int d^{2} q d \omega(-i \omega) u_{1}(\mathbf{q}, \omega) \sigma(-\mathbf{q},-\omega) .
$$

Using (3) and (4) we obtain

$$
\Delta E=(2 \pi)^{3} \int d^{2} q d \omega \frac{\omega K^{2} \operatorname{Im} M(\mathbf{q}, \omega)}{|1+K M(\mathbf{q}, \omega)|^{2}}\left\langle\left|u_{0}(\mathbf{q}, \omega)\right|^{2}\right\rangle,
$$

where we have performed an ensemble (or thermal) average denoted by $\langle.$.$\rangle . Next, note that$

$$
\left\langle\left|u_{0}(\mathbf{q}, \omega)\right|^{2}\right\rangle=\frac{A_{0} t_{0}}{(2 \pi)^{3}} C_{u u}(\mathbf{q}, \omega),
$$

where $A_{0}$ is the surface area, and

$$
C_{u u}(\mathbf{q}, \omega)=\frac{1}{(2 \pi)^{3}} \int d^{2} x d t\left\langle u_{0}(\mathbf{x}, t) u_{0}(0,0)\right\rangle e^{i(\mathbf{q} \cdot \mathbf{x}-\omega t)}
$$

is the displacement correlation function. We can also write

$$
C_{u u}(\mathbf{q}, \omega)=\int d^{2} q^{\prime} d \omega^{\prime}\left\langle u_{0}(-\mathbf{q},-\omega) u_{0}\left(\mathbf{q}^{\prime}, \omega^{\prime}\right)\right\rangle .
$$

Substituting (6) in (5) gives the heat current $J_{0 \rightarrow 1}=$ $\Delta E / A_{0} t_{0}$ from solid $\mathbf{0}$ to solid $\mathbf{1}$ :

$$
J_{0 \rightarrow 1}=2 \int d^{2} q \int_{0}^{\infty} d \omega \frac{\omega K^{2} \operatorname{Im} M(\mathbf{q}, \omega)}{|1+K M(\mathbf{q}, \omega)|^{2}} C_{u u}\left(\mathbf{q}, \omega ; T_{0}\right) .
$$

where $C_{u u}$ depends on the temperature $T_{0}$ of solid $\mathbf{0}$. A similar equation with $T_{0}$ replaced by $T_{1}$ gives the energy transfer from solid $\mathbf{1}$ to solid $\mathbf{0}$, and the net energy flow $J=J_{0 \rightarrow 1}-J_{1 \rightarrow 0}$.

With graphene in mind, we now assume that solid $\mathbf{0}$ is a membrane or two-dimensional (2D) system. We assume that $u_{0}(\mathbf{x}, t)$ satisfies

$$
\rho_{0} \frac{\partial^{2} u_{0}}{\partial t^{2}}=-\kappa \nabla^{2} \nabla^{2} u_{0}-\rho \eta \frac{\partial u_{0}}{\partial t}+f
$$

where $\kappa \approx 1 \mathrm{eV}[5]$ is the bending elasticity, $\eta$ a phenomenological friction coefficient, and $f(\mathbf{x}, t)$ a stochastic fluctuating force related to the temperature $T_{0}$ and the friction $\eta$ via the fluctuation-dissipation theorem:

$$
\left\langle f(\mathbf{q}, \omega) f\left(\mathbf{q}^{\prime}, \omega^{\prime}\right)\right\rangle=2(2 \pi)^{-3} \rho \eta k_{\mathrm{B}} T_{0} \delta\left(\omega+\omega^{\prime}\right) \delta\left(\mathbf{q}+\mathbf{q}^{\prime}\right) .
$$

where $\rho_{0}=n_{0} m_{0}$ is the mass density per unit area of the $2 \mathrm{D}$-system $\left(m_{0}\right.$ is the atom mass and $n_{0}$ the number of atoms per unit area). On the right hand side of (9) there should in principle be another term $K \nabla^{2} u_{0}$, due to the interaction with the substrate wall, but because of the assumed small magnitude of $K$, this term can be neglected for the relevant wavevectors $q$. The formalism above assumes that the system can be treated classically which is the case only if $\hbar \omega<<k_{\mathrm{B}} T_{0}$. If this condition is not satesfied one can take into account the most important quantum mechanical effects by replacing $k_{\mathrm{B}} T_{0}$ by $\Pi(\omega)=\hbar \omega\left[\exp \left(\hbar \omega / k_{\mathrm{B}} T_{0}\right)-1\right]^{-1 / 2}$.

From (9) we get

$$
u_{0}(\mathbf{q}, \omega)=\frac{f(\mathbf{q}, \omega)}{-\rho \omega^{2}+\kappa q^{4}-\rho \eta i \omega}
$$

Using (7) and (10) we get

$$
C_{u u}(\mathbf{q}, \omega)=\frac{2(2 \pi)^{-3} \rho \eta k_{\mathrm{B}} T_{0}}{\left|\rho \omega^{2}-\kappa q^{4}+\rho \eta i \omega\right|^{2}} .
$$

In the limit $\eta \rightarrow 0$ we get

$$
C_{u u}(\mathbf{q}, \omega)=\frac{\pi}{(2 \pi)^{3}} \frac{1}{\rho_{0} \omega_{1}^{2}} \delta\left(\omega-\omega_{1}\right),
$$

where $\omega_{1}=\left(\kappa / \rho_{0}\right)^{1 / 2} q^{2}=c(q) q$, where we have defined the velocity $c(q)=\left(\kappa / \rho_{0}\right)^{1 / 2} q$.

Substituting (11) in (8) and assuming weak coupling between the solids (i.e., $K$ is small), we get

$$
J_{0 \rightarrow 1}=\frac{k_{\mathrm{B}} T_{0}}{2 \pi \rho_{0}} \int_{0}^{\infty} d q \frac{q}{\omega_{1}} K^{2} \operatorname{Im} M\left(\mathbf{q}, \omega_{1}\right) .
$$

The heat transfer coefficient $\alpha=\left(J_{0 \rightarrow 1}-J_{1 \rightarrow 0}\right) /\left(T_{0}-T_{1}\right)$ is given by

$$
\alpha=\frac{k_{\mathrm{B}}}{2 \pi \rho_{0}} \int_{0}^{\infty} d q \frac{q}{\omega_{1}} K^{2} \operatorname{Im} M\left(\mathbf{q}, \omega_{1}\right) .
$$

Using the expression for $M(\mathbf{q}, \omega)$ derived in [4, 6] and $\omega_{1}=c(q) q$ gives

$$
\alpha=\frac{k_{\mathrm{B}} K^{2} \xi}{\rho_{0} \rho_{1} c_{\mathrm{T}}^{3}},
$$

where

$$
\begin{gathered}
\xi=\frac{1}{2 \pi} \int_{0}^{q_{\mathrm{c}}} d q \frac{1}{q} \frac{c(q)}{c_{\mathrm{T}}} \\
\times \operatorname{Re}\left(\frac{\left[\frac{c^{2}(q)}{c_{\mathrm{L}}^{2}}-1\right]^{1 / 2}}{\left[\frac{c^{2}(q)}{c_{\mathrm{T}}^{2}}-2\right]^{2}+4\left[\frac{c^{2}(q)}{c_{\mathrm{T}}^{2}}-1\right]^{1 / 2}\left[\frac{c^{2}(q)}{c_{\mathrm{L}}^{2}}-1\right]^{1 / 2}}\right),
\end{gathered}
$$

where $c_{\mathrm{L}}, c_{\mathrm{T}}$ and $\rho_{1}$ are the longitudinal and transverse sound velocities, and the mass density, respectively, of solid 1. The cut off wavevector $q_{\mathrm{c}} \approx \pi / a_{1}\left(a_{1}\right.$ is the lattice constant, or the average distance between two nearby atoms) of solid $\mathbf{1}$. 
There are two contributions to the integral $\xi$. One is derived from $c(q)>c_{\mathrm{L}}$, but for graphene on a- $\mathrm{SiO}_{2}$ studied below, this gives only $\sim 10 \%$ of the contribution to the integral. For $c(q)<c_{\mathrm{L}}$ the term after the Re operator is purely imaginary (and will therefore not contribute to the integral), except for the case where the denominator vanish. It is found that this pole-contribution gives the main contribution $(\sim 90 \%)$ to the integral, and corresponds to the excitation of a Rayleigh surface (acoustic) phonon of solid 1. This process involves energy exchange between a bending vibrational mode of the graphene and a Rayleigh surface phonon mode of solid $\mathbf{1}$. The denominator vanish when $c(q)=c_{\mathrm{R}}$ where

$$
\left[\frac{c_{\mathrm{R}}^{2}}{c_{\mathrm{T}}^{2}}-2\right]^{2}-4\left[1-\frac{c_{\mathrm{R}}^{2}}{c_{\mathrm{T}}^{2}}\right]^{1 / 2}\left[1-\frac{c_{\mathrm{R}}^{2}}{c_{\mathrm{L}}^{2}}\right]^{1 / 2}=0 .
$$

Note that the Rayleigh velocity $c_{\mathrm{R}}<c_{\mathrm{T}}$ but close to $c_{\mathrm{T}}$. For example, when $c_{\mathrm{L}} / c_{\mathrm{T}}=2, c_{\mathrm{R}} \approx 0.93 c_{\mathrm{T}}$, and the pole contribution to the integral in $\xi$ is 0.083 .

In the model above the heat transfer between the solids involves a single bending mode of the membrane or 2Dsystem. In reality there will always be some roughness at the interface which will somewhat blurre the wavevector conservation rule. We therefore expect some narrow band of bending modes to be involved in the energy transfer, rather than a single mode. Nevertheless, the model study above assumes implicitly that, due to lattice nonlinearity (and defects), there exist phonon scattering processes which rapidly transfer energy to the bending mode involved in the heat exchange with the substrate. This requires very weak coupling to the substrate, so that the energy transfer to the substrate is so slow that the bending mode can be re-populated by phonon scattering processes in the 2D-system, e.g., from the in-plane phonon modes, in such a way that its population is always close to what would be the case if complete thermal equilibrium occurs in the $2 \mathrm{D}$-system. This may require high temperature in order for multi-phonon scattering processes to occur by high enough rates.

Graphene, the recently isolated 2D-carbon material with unique properties due to its linear electronic dispersion, is being actively explored for electronic applications [7]. Important properties are the high mobilities reported especially in suspended graphene, the fact that graphene is the ultimately thin material, the stability of the carbon-carbon bond in graphene, the ability to induce a bandgap by electron confinement in graphene nanoribbons, and its planar nature, which allows established pattering and etching techniques to be applied. Recently it has been found that the heat generation in graphene field-effect transistors can result in high temperature and device failure [8]. Thus, it is important to understand the the mechanisms which influence the heat flow.

The graphene $-\mathrm{a}-\mathrm{SiO}_{2}$ interaction is probably of the

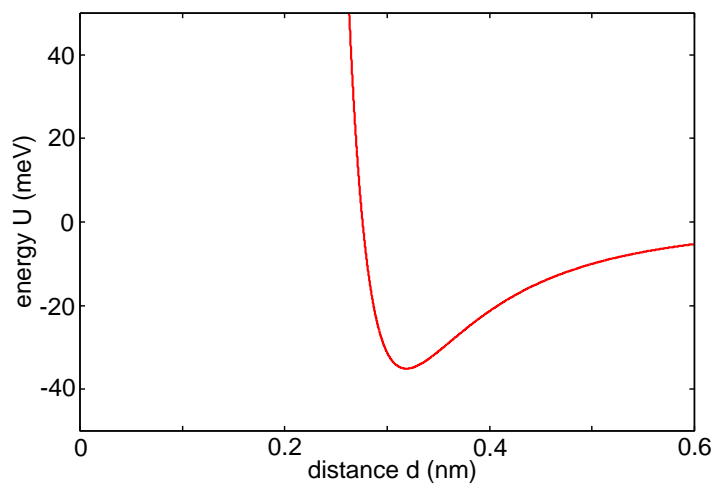

FIG. 2: The calculated graphene $-\mathrm{a}-\mathrm{SiO}_{2}$ interaction energy $U(d)$ per graphene carbon atom, as a function of the separation $d$ (in $\mathrm{nm}$ ) between the center of a graphene carbon atom and the center of the first layer of substrate atoms. See text for details.

van Der Waals type. In Ref. 9] the interaction between the graphene $\mathrm{C}$-atoms and the substrate $\mathrm{Si}$ and $\mathrm{O}$ atoms was assumed to be described by the Lennard-Jones (LJ) pair-potentials with different parameters. Here we use a simplified picture where the substrate atoms form a simple cubic lattice with the lattice constant determined by $a_{1}=\left(\bar{m} / \rho_{1}\right)^{1 / 3} \approx 0.25 \mathrm{~nm}$, where $\bar{m}=$ $\left(m_{\mathrm{Si}}+2 m_{\mathrm{O}}\right) / 3 \approx 3.32 \times 10^{-26} \mathrm{~kg}$ is the average substrate atomic mass, and $\rho_{1} \approx 2200 \mathrm{~kg} / \mathrm{m}^{3}$ the mass density of a-SiO ${ }_{2}$. We also use the effective LJ energy parameter, $\epsilon=\left(\epsilon_{\mathrm{Si}}+2 \epsilon_{\mathrm{O}}\right) / 3 \approx 5.3 \mathrm{meV}$, and the bondlength parameter $\sigma=\left(\sigma_{\mathrm{Si}}+2 \sigma_{\mathrm{O}}\right) / 3 \approx 0.31 \mathrm{~nm}$. With these parameters we can calculate the graphene- $-\mathrm{SiO}_{2}$ interaction energy, $U(d)$, per graphene carbon atom, as a function of the separation $d$ (in $\mathrm{nm}$ ) between the center of a graphene carbon atom and the center of the first layer of substrate atoms. We find (see Fig. 2) the graphene-a$\mathrm{SiO}_{2}$ binding energy $E_{\mathrm{b}}=-U\left(d_{\mathrm{eq}}\right) \approx 35 \mathrm{meV}$ per carbon atom, and the force constant $k=U^{\prime \prime}\left(d_{\mathrm{eq}}\right)$ (where $d_{\mathrm{eq}} \approx 0.32 \mathrm{~nm}$ is the equilibrium separation) $k=K a_{0}^{2}=$ $2.4 \mathrm{~N} / \mathrm{m}$ per carbon atom. This gives the perpendicular graphene - $\mathrm{a}_{-} \mathrm{SiO}_{2}$ (uniform) vibration frequency $\omega_{\perp} \approx\left(k / m_{0}\right)^{1 / 2} \approx 55 \mathrm{~cm}^{-1}$, which is similar to what is observed for the perpendicular vibrations of linear alkane molecules on many surfaces (e.g., about $55-60 \mathrm{~cm}^{-1}$ for alkanes on metals and on hydrogen terminated diamond $\mathrm{C}(111)[10])$. Using $K=k / a_{0}^{2}=1.82 \times 10^{20} \mathrm{~N} / \mathrm{m}^{3}$, and the transverse and longitudinal sound velocities of solid $1\left(c_{\mathrm{T}}=3743 \mathrm{~m} / \mathrm{s}\right.$ and $\left.c_{\mathrm{L}}=5953 \mathrm{~m} / \mathrm{s}\right)$, from (13) we obtain $\alpha \approx 3 \times 10^{8} \mathrm{~W} / \mathrm{Km}^{2}$.

The heat transfer coefficient between graphene and a perfectly flat $\mathrm{a}-\mathrm{SiO}_{2}$ substrate has not been measured directly, but measurements of the heat transfer between carbon nanotubes and sapphire by Maune et al[11] indicate that it may be of order $\alpha \approx 8 \times 10^{8} \mathrm{~W} / \mathrm{m}^{2} \mathrm{~K}$. This value was deduced indirectly by measuring the breakdown voltage of carbon nanotubes, which could be 
related to the temperature increase in the nanotubes. Molecular dynamics calculations [9] for nanotubes on a$\mathrm{SiO}_{2}$ gives $\alpha \approx 3 \times 10^{8} \mathrm{~W} / \mathrm{m}^{2} \mathrm{~K}$ (here it has been assumed that the contact width between the nanotube and the substrate is $1 / 5$ of the diameter of the nanotube). Finally, using a so called $3 \omega$ method, Chen et al 12 have measured the heat transfer coefficient $\alpha \approx$ $2 \times 10^{8} \mathrm{~W} / \mathrm{m}^{2} \mathrm{~K}$.

As pointed out in Sec. 3, the model developed above for the heat transfer involves a single, or narrow band, of bending modes of the membrane or 2D-system. In order for this model to be valid, the coupling to the substrate must be so weak that the energy transfer to the substrate from the bending mode occur so slowly that the mode can be re-populated by phonon scattering processes, in such a way that its population is always close to what is expected if full thermal equilibrium would occur within the 2D-system. This may require high temperature in order for multi-phonon scattering processes to occur by high enough rates. We suggest that this may be one reason for the decrease in the heat transfer coefficient observed for the graphene - a- $\mathrm{SiO}_{2}$ system below room temperature [12].

In Ref. 8] the temperature profile in the graphene under current was studied experimentally. The heat transfer coefficient between graphene and the a-SiO $\mathrm{S}_{2}$ substrate was determined by modeling the heat flow using the standard heat flow equation with the heat transfer coefficient as the only unknown quantity. The authors found that using a constant (temperature independent) heat transfer coefficient $\alpha \approx 2.5 \times 10^{7} \mathrm{~W} / \mathrm{m}^{2} \mathrm{~K}$ the calculated temperature profiles in graphene are in good agreement with experiment. This $\alpha$ is at least 10 times smaller than expected for perfectly flat surfaces (see above). In an earlier paper [13] we have studied the heat transfer between graphene and $\mathrm{a}-\mathrm{SiO}_{2}$. In that study we assumed that because of surface roughness the graphene only makes partial contact with the $\mathrm{SiO}_{2}$ substrate, which will reduce the heat transfer coefficient as compared to the perfect contact case. We showed how observed heat transfer can be explained as resulting from the spreading resistance, rather than the heat resistance from the area of real contact.

It has recently been suggested 14] that the heat transfer between graphene and a- $\mathrm{SiO}_{2}$ may involve photon tunneling [15]. That is, coupling via the electromagnetic field between electron-hole pair excitations in graphene and optical phonons in a-SiO${ }_{2}$. However, our calculations indicate that for graphene adsorbed on $\mathrm{a}-\mathrm{SiO}_{2}$ the field coupling gives a negligible contribution to the heat transfer [13].

To summarize, we have studied the heat transfer between weakly coupled systems with flat interface. We have presented simple analytical results which can be used to estimate the heat transfer coefficient. Detailed results was presented for the heat transfer between a membrane and a semi-infinite solid. For this case the energy transfer is dominated by energy exchange between a bending vibrational mode of the graphene, and a Rayleigh surface phonon mode of the substrate. This model assumes implicitly that, due to lattice nonlinearity (and defects), there exist phonon scattering processes which rapidly transfer energy to the bending mode involved in the heat exchange with the substrate. This may require high temperature in order for multi-phonon scattering processes to occur at high enough rate. As an application we have considerd the heat transfer between graphene and amorphous $\mathrm{SiO}_{2}$. The calculated value of the heat transfer coefficient was found to be in good agreement with the value deduced from the experimental data.

We thank P. Avouris for drawing our attention to Ref. [8]. B.N.J.P. was supported by Invitation Fellowship Programs for Research in Japan from Japan Society of Promotion of Science (JSPS). H.U. was supported by the Grant-in-Aid for Scientific Research B (No. 21310086) from JSPS.

[1] B.N.J. Persson, Surf. Sci. Rep. 61, 201 (2006).

[2] B.N.J. Persson, B. Lorenz and A.I. Volokitin, The European Physics Journal E31, 3 (2010).

[3] E.T. Swartz and R.O. Pohl, Rev. Mod. Phys. 61, 605 (1989).

[4] B.N.J. Persson, Journal of Chemical Physics 115, 3840 (2001).

[5] A. Fasolino, J.H. Los and M.I. Katsnelson, NATURE 6, 858 (2007).

[6] B.N.J. Persson and R. Ryberg, Phys. Rev. B32, 3586 (1985).

[7] A.K. Geim and K.S. Novoselov, Nat. Mater. 6, 183 (2007).

[8] M. Freitag, M. Steiner, Y. Martin, V. Perebeinos, Z. Chen, J.C. Tsang and P. Avouris, Nano Letters 9, 1883 (2009).

[9] Z-Y Ong and E. Pop, Phys. Rev. B81, 155408 (2010).

[10] M. Fuhrmann and Ch. Wöll, New Journal of Physics 1, 1 (1998).

[11] H. Maune, H-Y Chiu and M. Bockrath, Applied Physics Letter 89, 013109 (2006).

[12] Z. Chen, W. Jang, W. Bao, C.N. Lau and C. Dames, Applid Physics Letters 95, 161910 (2009).

[13] B.N.J. Persson and H. Ueba, subm. to Nano Letters.

[14] S. V. Rotkin, V. Perebeinos, A.G. Petrov and P. Avouris, Nano Letters 9, 1850 (2009); M. Freitag, M. Steiner, Y. Martin, V. Perebeinos, Z. Chen, J.C. Tsang and P. Avouris, Nano Letters 9, 1883 (2009).

[15] A.I. Volokitin and B.N.J. Persson, Reviews of Modern Physics 79, 1291 (2007). 